\title{
Beyond Rules-of-Thumb: Optimizing EPMA Measurements
}

\author{
Nicholas W. M. Ritchie ${ }^{1 *}$ \\ 1. National Institute of Standards and Technology, Material Measurement Science Division, \\ Gaithersburg, MD, USA. \\ * Corresponding author: nicholas.ritchie@nist.gov
}

Determining the conditions for the optimal EPMA measurement is a challenge. Rules-of-thumb are commonly used to guide the selection of a set of standard materials, the beam energy $\left(\mathrm{E}_{0}\right)$, suitable sample preparation and other measurement parameters. However, these rules are ambiguous and sometime contradictory and, while better than nothing, don't necessarily produce the optimal results. Some of the common rules-of-thumb include "maintain an overvoltage of two", "K-lines are more accurate than L- or M-lines," "measurements based on characteristic x-rays with energies less than 1 $\mathrm{keV}$ are typically less accurate," "use a standard similar to the unknown to minimize matrix correction inaccuracies," and "maintain a high enough beam energy to avoid surface influences like contamination and oxide layers." Notice that even this small set of rules-of-thumb are mutually contradictory. For years, we've accepted this situation and accepted that make the best measurements using EPMA is an art that can be mastered with many years of experience. We contend that there is a better way - use uncertainty calculations to optimize measurement conditions. The first necessary step is the ability to calculate an exhaustive uncertainty model.

Historically, the uncertainty budget has been limited to considerations that limited the precision of the measurement (count statistics, dose stability)[1]. In 2012, we extended the understanding to include terms related to x-ray absorption and electron backscatter[2]. More recently, we've extended the uncertainty model to consider many more factors including measurement conditions, sample preparation (surface roughness and coating layers), standard and unknown composition and model parameters. This new model gives us a more detailed mechanism to understand how our measurement choices influence results. For example, how smooth does a sample/standard's sample need to be to make a negligible contribution? How consistent does a conductive coating have to be between standard and unknown? Also instrumental conditions: How well do we have to control the sample orientation? What material represents the best standard? How well do we have to control the incident beam energy through both the instrument setting but also surface charge control? Beam energy is one of the critical parameters we can control but determining the optimal beam energy is subtle based on the interplay of the elements to be measured, the choice of characteristic lines, the choice of standards and other measurement choices.

Consider the engineered glass, K240, consisting of the elements O (24\%), Mg (3\%), Si (19 \%), Zn (4 $\%), \mathrm{Zr}(7 \%)$ and $\mathrm{Ba}\left(27 \%\right.$ by mass). For most of the elements, the $\mathrm{K}-\mathrm{L}_{3}$ line is chosen except for $\mathrm{Zr}$ and $\mathrm{Ba}$, in which cases, the $\mathrm{L}_{3}-\mathrm{M}_{5}$ line was selected. The $\mathrm{Zn} \mathrm{K}$ shell edge is at approximately $10 \mathrm{keV}$ so we considered beam energies from $12 \mathrm{keV}$ to $30 \mathrm{keV}$. In this example, we assumed that the beam energy was controlled to within $\pm 100 \mathrm{eV}$, the samples were smooth to within $10 \mathrm{~nm}$, the take-off angle was controlled to within $\pm 0.5^{\circ}$ and the k-ratios were equivalent to a $60 \mathrm{nA} \cdot \mathrm{s}$ dose measured on 0.1 steradian SDD. Figure 1 show the results of two uncertainty calculations which differ only in the standards used to perform the measurement. A), B) and C) represent simple standards $\mathrm{MgO}$ for $\mathrm{O}, \mathrm{MgSi}_{2} \mathrm{O}_{5}$ for $\mathrm{Ba}$ and pure elements for the remaining elements. D), E) and F) represents the (somewhat artificial) situation of a standard of identical composition to the unknown. The two extreme sets of standards are designed to 
exemplify how different portions of the uncertainty budget dominate under different assumptions. For a low energy $\mathrm{x}$-ray like $\mathrm{O} \mathrm{K}-\mathrm{L}_{3}$, with a simple standard, the mass absorption coefficient dominates with surface roughness also a significant contribution. As the beam energy increases, the absorption correction uncertainty increases as the mean exit path length increases. In contrast, when an identical standard is used, the surface roughness dominates and the absorption correction becomes negligible. Because the $\mathrm{O} K-\mathrm{L}_{3}$ line is relatively intense, the uncertainty in the k-ratio is never a dominant contribution. For $\mathrm{Si} \mathrm{K}-\mathrm{L}_{3}$ using $\mathrm{Si}$ as a standard, the overall uncertainty budget is minimized near 14 $\mathrm{keV}$ as the uncertainty due to the beam energy decreases and due to the absorption correction increases. With an identical standard, the overall uncertainty varies less and reaches a minimum nearer $18 \mathrm{keV}$. For $\mathrm{Zn} \mathrm{K- \textrm {L } _ { 3 }}$ using $\mathrm{Zn}$ as a standard, the overvoltage is very low at $10 \mathrm{keV}$ leading to a sensitivity to beam energy and a small x-ray intensity leading to high uncertainty in the k-ratio. As the incident beam energy increases, the overall uncertainty plummets. Similar behavior is seen with the identical standard, however the uncertainty is actually larger at low beam energies because of the small quantity of $\mathrm{Zn}$ in the standard and unknown and the low overvoltage. In contrast to $\mathrm{O}$ and $\mathrm{Si}$, an identical standard can actually be worse than a pure element standard when element is present in small amounts. These calculations demonstrate that there is no single optimal beam energy for this measurement. The analyst must ultimately make a judgement call based on the intended use of the results to determine suitable measurement conditions.

\section{References:}

[1] R Marinenko and S Leigh, IOP Conf Series: Mat Science and Engineering 7(1) (2010), p. 012017.

[2] N Ritchie and D Newbury, Analytical Chemistry 84 (2012), p. 9956.
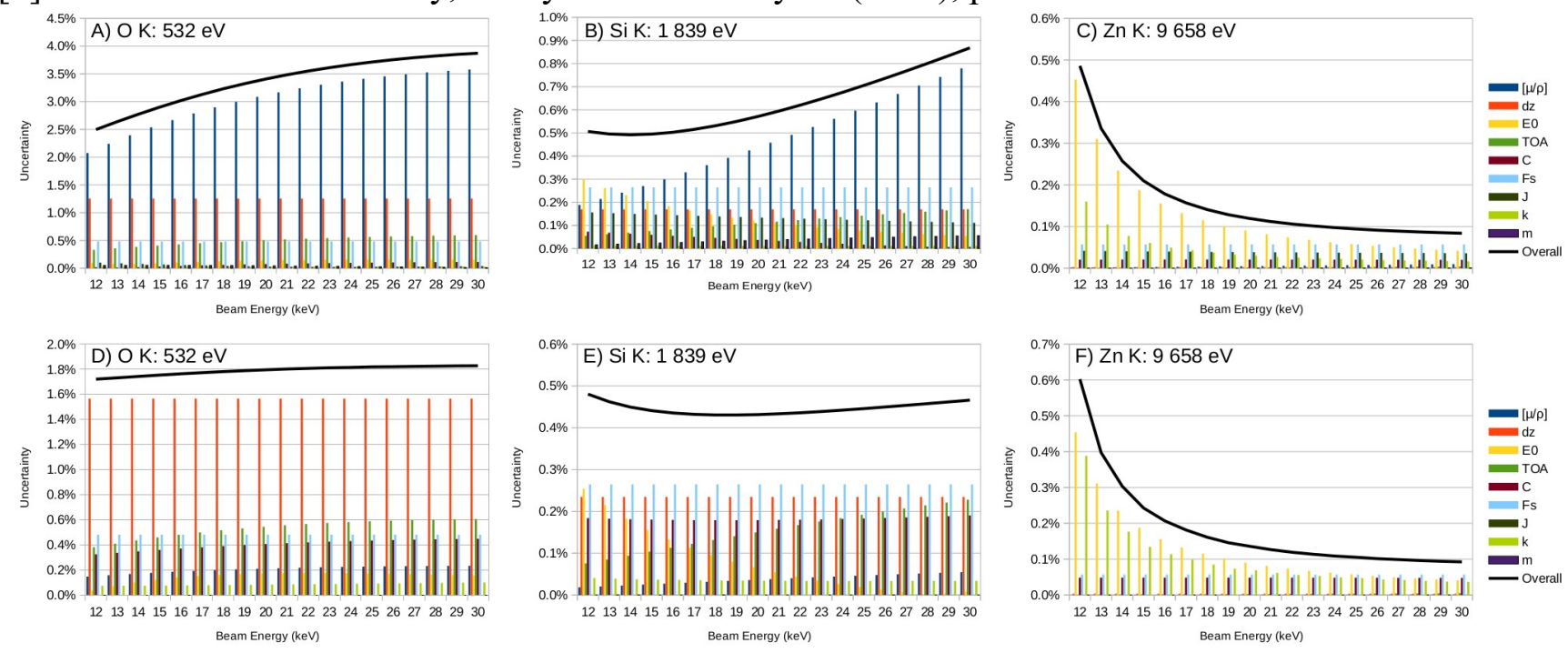

Figure 1. Comparing the uncertainty budgets for three elements $\mathrm{O}$, Si and $\mathrm{Zn}$ measured using the $\mathrm{K}-\mathrm{L}_{3}$ line over a range of incident beam energies from $12 \mathrm{keV}$ to $30 \mathrm{keV}$. A), B), and C) represent simple standards and D), E), and F) represent using an identical standard. The sources of uncertainty are the mass absorption coefficient, $[\mu / \rho]$, the surface roughness, $\mathrm{dz}$, the beam energy, E0, the take-off angle, TOA, the composition of the standard and unknown, $\mathrm{C}$, the mean ionization potential, J, the k-ratio, $\mathrm{k}$, and the ionization cross-section, $\mathrm{m}$. The black 'Overall' line represents the total uncertainty budget (the sum in quadrature of the independent contributions.) 